\title{
Cerium-Doped Endohedral Fullerene: A Density-Functional Theory Study
}

\author{
A. Z. AlZahrani \\ Physics Department, Faculty of Science, King Abdulaziz University, P.O. Box 80203, Jeddah 21589, Saudi Arabia \\ Correspondence should be addressed to A. Z. AlZahrani, azalzahrani@kau.edu.sa
}

Received 15 February 2012; Accepted 29 March 2012

Academic Editors: C. Andreani, A. Cantarero, and A. Krimmel

Copyright ( 2012 A. Z. AlZahrani. This is an open access article distributed under the Creative Commons Attribution License, which permits unrestricted use, distribution, and reproduction in any medium, provided the original work is properly cited.

First-principles total energy calculations of the structural and electronic properties of Ce-doped fullerene have been performed within the framework of the density functional theory at the generalized gradient approximation level. Among various locations, $\mathrm{Ce}$ atom was found to engage with the six-fold carbon ring. The total energy is found to significantly change as the Ce atom being shifted from the center of the cage toward the edge close to the six-membered ring where the total energy reaches its local minimum. Moreover, repulsive interaction between Ce atom and the cage components turns as the adatom directly interacts with the six $\mathrm{C}$ atoms of the ring. The lowest-energy $\mathrm{CeC}_{60}$ geometry is found to have a binding energy of approximately $5.34 \mathrm{eV}$, suggesting strong interaction of the dopant with the cage members. Furthermore, fundamental key structural parameters and the total density of states of the optimized structure have been determined and compared with the available data.

\section{Introduction}

Nowadays, the interest in nanoscale materials has rapidly increased due to their positive use in various technical and scientific researches relating on the energy, environment, and biomedical fields. Carbon is one of the most amazing elements with a vast range of exotic properties. These properties have shed the light on carbon-based nanostructures, such as fullerenes [1], carbon nanotubes [2], and graphene [3]. Since its discovery in 1985 (Nobel Prize in 1996) [1], the subject of fullerene molecule $\mathrm{C}_{60}$, the closed-cage carbon molecules, has attracted immense interest and attention due to its fascinating chemical and physical properties [4-7]. Indeed, the discovery of fullerenes has opened the gate for the production and investigation of much more suitable material building blocks which could be used as drug deliverers in medical applications and/or possible superconductors in technological applications. Therefore, fullerene provides an entirely new branch of chemistry, materials science, and physics. Moreover, solid fullerene is treated as a semiconductor material [8], and its structure is described in the face-centered cubic lattice. Unlike the carbon nanotubes with only hexagonal cells, the fullerene consists of both pentagons and hexagons with two classes of bond lengths: a shorter bond length connecting two successive hexagons and a longer bond length joining a hexagon and pentagon. While the dependence of properties at nanoscale provides an opportunity to test and experiment with various materials, the fullerene is considered as a suitable candidate for nanotechnology since it has the right size and chemical stability. Therefore, the properties of fullerenes can be tuned and enhanced by doping various elements. Depending on the site of doping, fullerenes can be functionalized into three main categories: exohedral fullerenes with the dopant outside the cage, endohedral fullerenes with the dopant inside the cage, and on-site fullerene with the dopant replacing one $\mathrm{C}$ atom.

Recently, endohedral fullerene, a member of fullerene family with a dopant encaged within the sphere, has received remarkable attention in various experimental [9-18] and theoretical [19-30] investigations due to its exotic physical and chemical properties such as pseudoatom behavior, magnetism, and superconductivity. The incorporation of lanthanum (La) atom inside the cage of the $\mathrm{C}_{60}$ structure has been experimentally investigated by Heath et al. [9] to detect the existence of endohedral metallofullerene complexes. They found, using laser vaporization and mass spectra, that 
both $\mathrm{La}$ and $\mathrm{La}_{2}$ form endohedral fullerenes. Later, Shinohara and coworkers have studied the production of yttrium endohedral $\mathrm{YC}_{60}[10]$ and scandium endohedral $\mathrm{ScC}_{60}$ [11] using arc and laser vaporization of metal-graphite composites in helium atmosphere. Kortan et al. [12] found that a high concentration of calcium $(\mathrm{Ca})$ doped $\mathrm{C}_{60}$ experienced phase transitions from face-centered cubic to simple cubic via body-centered cubic. Furthermore, doping of radioactive elements, such as beryllium (Be) [13] and gadolinium (Gd) [14], has been found to be properly encaged inside a $\mathrm{C}_{60}$ cluster, leading to substantial applications in nuclear wasting disposal such as nanocontainer radioactivities [31]. Other experimental studies have been reported on the purification of $\mathrm{EuC}_{60}$ [15] and $\mathrm{ErC}_{60}$ [16] using chemical extraction from carbon soot and high-performance liquid chromatography (HPLC) and investigated the optical properties of purified $\mathrm{LiC}_{60}$ [17] using Raman and infrared spectra. Theoretically, the electronic and optical properties of potassium-(K-) doped fullerene $[19,20]$ have been calculated using the local density approximation (LDA) of the density functional theory (DFT). In their study, Xu et al. [20] have reported that face-centered cubic $\mathrm{K}_{3} \mathrm{C}_{60}$ has a superconducting nature, whereas body-centered cubic $\mathrm{K}_{6} \mathrm{C}_{60}$ behaves like an insulator with sufficient agreement with the electron-energy-loss spectra measurements. Saito and Oshiyama [21] studied the electronic structure of $\mathrm{Ca}_{3} \mathrm{C}_{60}$ and $\mathrm{Ca}_{5} \mathrm{C}_{60}$ solids. Their calculations revealed electronic charge transfer from $\mathrm{Ca} 4 \mathrm{~s}$ states to the lowest-unoccupied state $\left(t_{1 \mathrm{u}}\right)$ of the $\mathrm{C}_{60}$ cluster and a strong hybridization between the second lowestunoccupied state $\left(t_{1 \mathrm{~g}}\right)$ of the $\mathrm{C}_{60}$ cluster and the $4 \mathrm{~s}$ states of the Ca atoms at the off-center position of the octahedral interstitial site. Thereafter, Umemoto and Saito [22] investigated the electronic properties of the superconducting bodycentered orthorhombic barium fullerene $\mathrm{Ba}_{4} \mathrm{C}_{60}$ within the framework of the LDA of the DFT scheme. It has been found that $\mathrm{Ba}$ states strongly hybridize with $\mathrm{C}_{60}$ states through pentagons faces, leading with the asymmetric lattice to the splitting of the states, and hence, $t_{1 \mathrm{~g}}$ is partially filled. Lu and coworkers [23] have presented $a b$ initio investigations for the structural and electronic properties of endohedral beryllium $\mathrm{BeC}_{60}$ using the DFT method. Their results show that Be occupies the center of the fullerene cage preserving its atomic electronic configuration. They also found a repulsive interaction between the $\mathrm{Be}$ atom and the fullerene cage. Moreover, it has been found that small hydrogenated atoms can also be doped in fullerene. Erkoç and Türker [2426] have reported, using semiempirical self-consistent field molecular orbital (SCF-MO) method, on the structural and electronic properties of fullerene upon the deposition of hydrogenated $\mathrm{B}, \mathrm{C}, \mathrm{N}, \mathrm{Al}, \mathrm{Si}$, and $\mathrm{P}$ atoms. It has been found that these endofullerene are stable but endothermic. Using $a b$ initio molecular dynamics simulations based on the allelectron mixed-basis approach, Ohtsuki and Ohno [27] have shown that the inclusion of a Po atom into $\mathrm{C}_{60}$ through either pentagon or hexagon rings is equally preferable. Very recently, Yang et al. [7] have presented first-principles DFT calculations for the electronic structure of single B-, N-, Co-, $\mathrm{P}-$, and Bi-doped $\mathrm{C}_{60}$ solids. Also they have reproduced the calculations for two and three $\mathrm{Bi}$ atoms in $\mathrm{C}_{60}$ solids.
For a single dopant concentration, they have found that $\mathrm{B}-, \mathrm{P}-$, and Co-doped $\mathrm{C}_{60}$ solids are $n$-type semiconductors, whereas $\mathrm{N}$ doped $\mathrm{C}_{60}$ solid is a $p$-type semiconductor. For two and three $\mathrm{Bi}$ atoms in $\mathrm{C}_{60}$ solids, the resultant structures are a $p$-type semiconductor and metal, respectively. Despite much available experimental and theoretical investigations of endohedral fullerenes, there is a lack of theoretical studies of endohedral fullerene doped by rare earth metals with unfilled $f$ shells. In the present work, we will report on the structural and electronic properties of Ce-doped $\mathrm{C}_{60}$ solids using DFT.

\section{Theoretical Framework}

Density-functional theory calculations employing the selfconsistent Kohn-Sham functional were performed using the Vienna $a b$ initio simulation package (VASP) [32-35]. The single-particle Kohn-Sham wave functions [36] were expanded using plane waves basis sets up to cut off energy of $20 \mathrm{Ry}$. The charge density cutoff energy was set to $80 \mathrm{Ry}$. We treated the electron-ion interactions using the ultrasoft pseudopotential [37]. The Perdew-Burke-Ernzerhof exchangecorrelation scheme [38] was considered to take into account the electron-electron interactions. Self-consistent solutions of the Kohn-Sham equations were obtained by employing a $2 \times 2 \times 2$ Monkhorst-Pack [39] grid of k-points for the integration over the Brillouin zone. The full electronic density of states was also calculated with a high $\mathbf{k}$-point sampling of $10 \times 10 \times 10$. The relative tolerance in the density matrix, taken as self-consistent field (SCF) convergence criterion, was set to $10^{-5} \mathrm{eV}$. Relaxed atomic positions were obtained by using the total energy and force minimization methods. The force tolerance in the minimization procedure was set to approximately $0.005 \mathrm{eV} / \AA$, in which no explicit symmetry constraints were imposed during the minimization.

\section{Results and Discussion}

The structural and electronic properties of the fcc fullerene have been determined to thoroughly investigate the modification of the fullerene upon the doping of Ce atom. Before presenting results for fullerene and Ce-doped fullerene, energy convergence tests with respect to the lattice constant, kinetic energy cut off, and special k-points were performed. Our calculations indicate that a minimum total energy value is obtained for a lattice parameter of $27.03 \operatorname{Bohr}(14.30 \AA)$ as shown in Figure 1. This value is slightly overestimated comparing with the experimental value of $14.17 \AA[40,41]$ but is in good agreement with previously reported value of $14.29 \AA$ along the $\mathrm{D}_{3 \mathrm{~d}}$ orientation [42]. In their study, Zólyomi et al. [42] have shown that the orientational effects play a significant role in determining the lattice constant of $\mathrm{C}_{60}$-based molecular crystals.

It is also clear from Figure 2 that very well-converged total energy values can be achieved for energy cutoff values of 20 Ry or higher.

Similarly, the choice of 6 special k-points (within $2 \times$ $2 \times 2$ Monkhorst-Pack grid) for self-consistent calculation of charge density is also found to be quite acceptable. Increasing the special k-points to 24 (within $4 \times 4 \times 4$ Monkhorst-Pack 


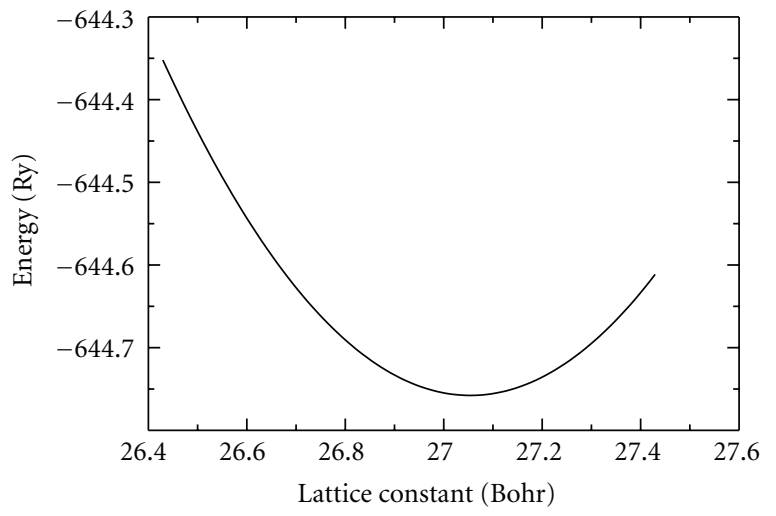

FIGURE 1: Energy convergence test to obtain the lattice parameter of fullerene.

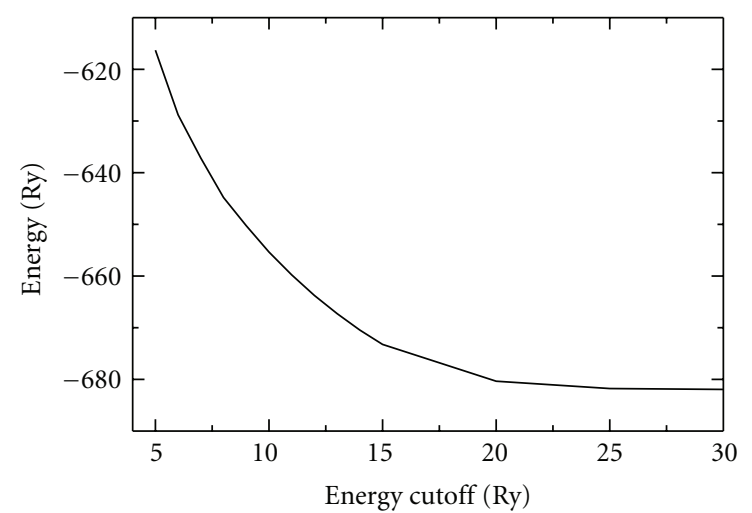

FIGURE 2: The variation of total energy as a function of the energy cutoff.

grid) only decreases the total energy by $0.02 \mathrm{eV} /$ cell. Figure 3 shows the relaxed geometry of the $\mathrm{C}_{60}$ configuration. It is found that the bond length of the hexagon-pentagon (6-5) is approximately $\mathrm{C}_{1}-\mathrm{C}_{2}=1.465 \AA$, while the $\mathrm{C}_{3}-\mathrm{C}_{4}=1.41 \AA$ for a hexagon-hexagon (6-6), suggesting quite agreement with the neutron diffraction measurements [43] of 1.46 and $1.39 \AA$, respectively.

These values are in the range of the double of the covalent radius of the carbon atom. Moreover, it is quite identical to the calculated $\mathrm{C}-\mathrm{C}$ bond length of diamond $(1.52 \AA)$ and graphite $(1.42 \AA)$. This, therefore, indicates wide range of similarity in the structural behavior of fullerene with the other C-based compounds. Furthermore, the inner and outer diameters of the ball are found to be 3.52 and $10.28 \AA$, respectively, indicating a mean ball diameter of $6.9 \AA$. These values are quite close to the previously reported values.

To investigate the fundamental electronic properties of fullerene, we have performed the electronic density of states (DOS) for fcc $\mathrm{C}_{60}$ as depicted in Figure 4. It is clearly shown that the energy band is approximately $1.57 \mathrm{eV}$, which is larger than the calculated band gap of $1.34 \mathrm{eV}$ obtained by Ching et al. [19]. Furthermore, it is noted that both the valence states and the conduction states consist of a number of small fragments. The origin of the occupied peaks is mainly due to the $p$ states of the $\mathrm{C}$ atoms with little contributions from

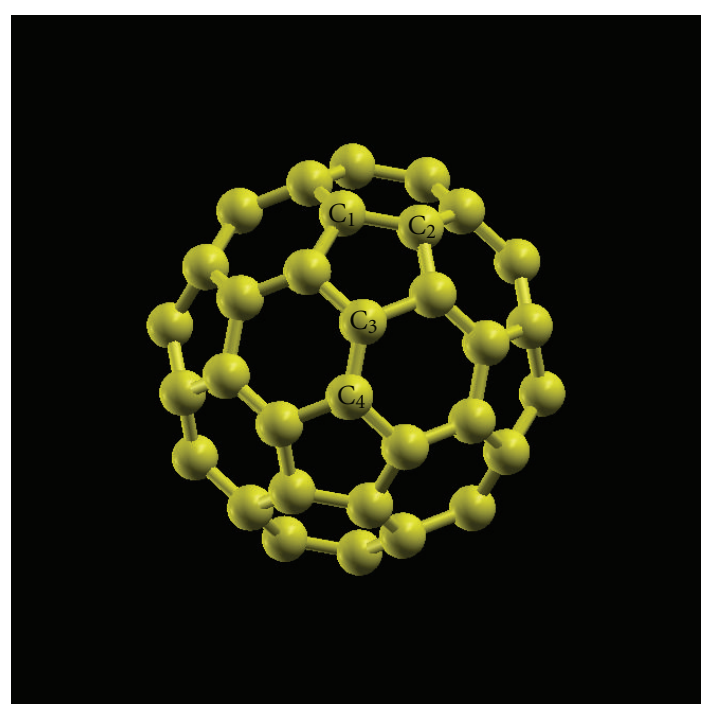

Figure 3: The equilibrium atomic geometry of $\mathrm{C}_{60}$ showing the pentagons and hexagons of the fullerene.

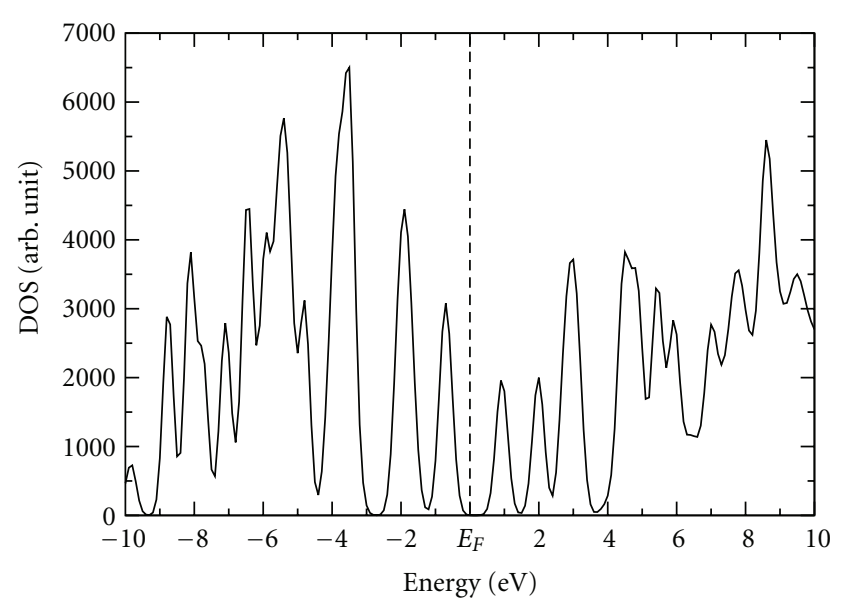

FIgure 4: The calculated electronic density of states (DOS) of $\mathrm{C}_{60}$. The Fermi level set at zero energy is represented by a vertical dashed line.

the carbon $s$ orbitals. These filled states are quite narrow and separated by very small gaps. Such a form of localized states is a fundamental feature of molecular-like structures.

The total charge density plot of the relaxed atomic structure of fullerene is plotted in a [110] plane passing through the center of fullerene, as shown in panel (a) of Figure 5. It is clearly shown that the charge is entirely accommodated around the five- and six-folded carbon atoms, indicating that fullerene has a similar charge distribution as the graphite and diamond structures with a $s p^{3}$ covalent bond formation. Moreover, the partial charge density plots of the highest occupied and lowest unoccupied states of the fullerene are also presented in panels (b) and (c) of Figure 5, respectively. These planar plots are performed along the [110] plane at the $\Gamma$ point of the Brillouin zone with an origin being chosen at the center of the ball. Figure 5 (b) shows that the highest occupied states mainly originate from the $p_{z}$-orbitals of the six-folded carbon atoms. The lowest unoccupied states 


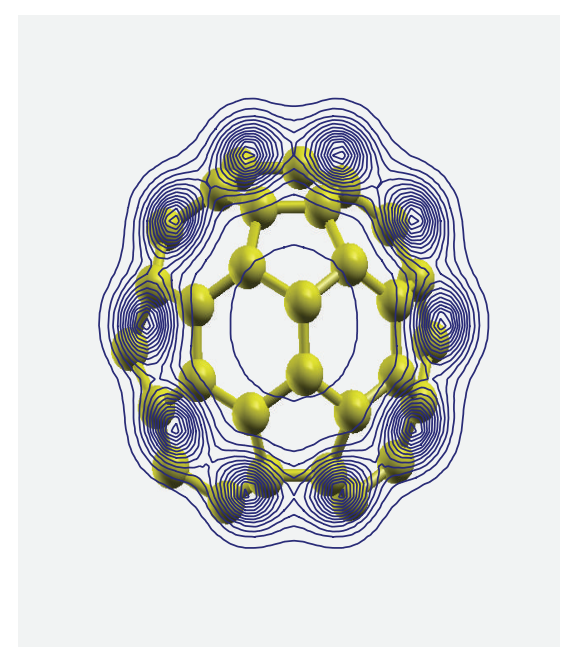

(a)

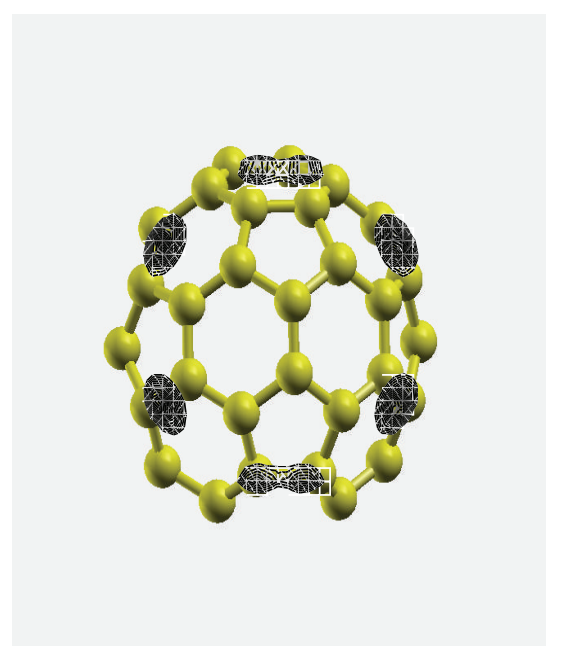

(b)

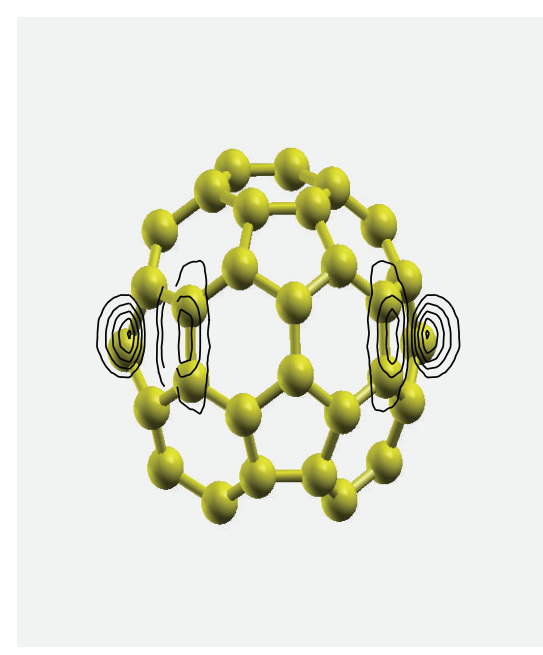

(c)

Figure 5: (a) Total charge density plot along the [110] plane passing through the center of the fullerene. Partial charge density plots at the $\Gamma$ point of the principle directions of the (b) highest occupied and (c) lowest unoccupied states. The minimum and maximum of charge density is 0.02 and $0.08 \mathrm{e} / \mathrm{a} . \mathrm{u}^{3}$, respectively.

are largely $p$ nature around the six-folded carbon atoms as shown in (c).

Having discussed the fundamental properties of the clean fullerene, we now are ready to investigate the effect of incorporation of $\mathrm{Ce}$ atom in the fullerene. To the best of our knowledge, the modification of the structural and electronic properties of $\mathrm{C}_{60}$ fullerene upon the doping of $\mathrm{Ce}$ atom is not yet established. So far, there is no direct information about the exact geometry of Ce-doped fullerene, and we have used our $a b$ initio density functional calculations to optimize the adsorption site of a single Ce adatom on the fullerene unit cell. To achieve such a purpose, five different initial positions taking into account all the symmetry sites and spanning uniformly the unit cell have been investigated, as shown in Figure 6. These doping sites are based on a single Ce atom placed (a) at the origin, (b) in a direct interact with the hexagon, (c) in a direct interact with the pentagon, (d) substituting one carbon atom, and (e) above the common C$\mathrm{C}$ bond connecting two consecutive-hexagon and pentagon.

Unlike the encapsulated $\mathrm{N}$ and noble gas atoms [44-47] and metallic atoms like Be, Ca, Li, Y, La, and Sc [23, 48-51], which all occupy the center or slightly positioned off-center of the fullerene, the absolute minimum corresponding to the lowest total energy has been found for Ce interacting directly with the six-folded ring of the fullerene, as indicated in panel (b) of Figure 6. It is also noted that during the optimization process, the $\mathrm{Ce}$ atom that was initially placed elsewhere inside the cage migrates to the six-membered ring. Our total energy calculation suggests that the total energy significantly changes as the Ce atom is shifted from the center of the cage toward the edge close to the hexagonal ring where the total energy reaches its local minimum, as shown in Figure 7. The interaction between $\mathrm{Ce}$ and the cage turns to be repulsive as the Ce atom directly interacts with the $\mathrm{C}$ atoms of the ring.

However, this optimal structure indicates that the $\mathrm{CeC}_{60}$ in its $\mathrm{C}_{2 \mathrm{v}}$ symmetry is the ground state. The binding energy of such a structure is determined to be $5.34 \mathrm{eV}$, which is smaller than the value obtained for $\mathrm{CeC}_{82}$ of $6.696 \mathrm{eV}$. However, this value is greater than the values of 2.65, 3.77, and $4.29 \mathrm{eV}$ obtained for $\mathrm{Gd}, \mathrm{La}$, and $\mathrm{PoC}_{60}$, respectively. The calculated value of binding energy indicates, though it is smaller than that of $\mathrm{CeC}_{82}$, a strong interaction of Ce $d$ and $f$ states with the $p$ states of the $\mathrm{C}$ atoms. With respect to the fully relaxed pure fullerene, the optimized hexagonhexagon and hexagon-pentagon $\mathrm{C}-\mathrm{C}$ bond lengths of the $\mathrm{CeC}_{60}$ are found to be slightly elongated by an approximately 0.0015 and $0.002 \AA$, respectively. These observations have also been seen in some metallic dopant in fullerene (see, e.g., $[22,23])$, suggesting possible charge transfer from the Ce atom to the $\mathrm{C}$ atoms. Despite that the structural change of the $\mathrm{CeC}_{60}$ is only a few decimals, it is vital to the ground state energy calculation. The average $\mathrm{Ce}-\mathrm{C}$ bond length is calculated to be $2.52 \AA$, which is almost equivalent to the sum of their covalent radii, indicating a strong bond formation. Consistent with the values obtained for pure fullerene, the inner and outer radii of the cage remain unaltered, suggesting that Ce does not distort the overall structure of the ball but a little appreciable elongation in the double and single $\mathrm{C}-\mathrm{C}$ bond of the cage rings as discussed earlier. Finally, we have calculated the average $\mathrm{C}-\mathrm{Ce}-\mathrm{C}$ bond angle of approximately $56.12^{\circ}$. It has also been calculated for $\mathrm{NiC}_{60}$ [52] that the drop shape is further characterized by the deviation from planarity of approximately $56.8^{\circ}$.

The electronic band structure of the endohedral Cedoped fullerene along the symmetry direction of the facecentered cubic Brillouin zone is depicted in Figure 8. At the $\Gamma$ point, it is clearly shown that the energy gap of the pure fullerene has been significantly reduced to approximately $0.2 \mathrm{eV}$ due to the quite strong interaction between the dopant and the carbon species. The highest occupied state slightly crosses the Fermi level at both $\mathrm{L}$ and $\mathrm{K}$ points of the Brillouin zone. We have noticed that the highest occupied state is quite 


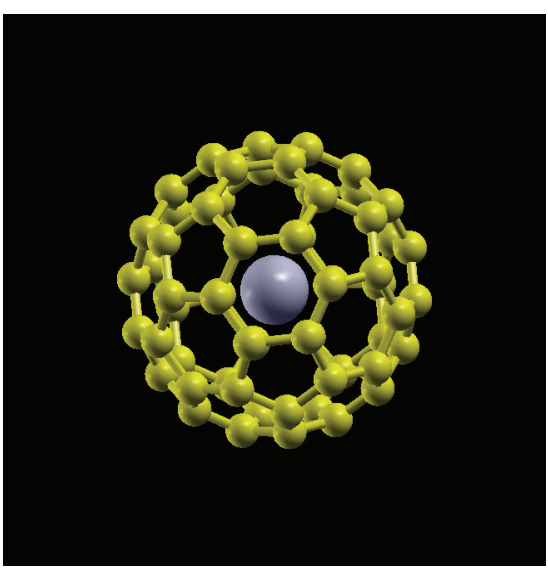

(a)

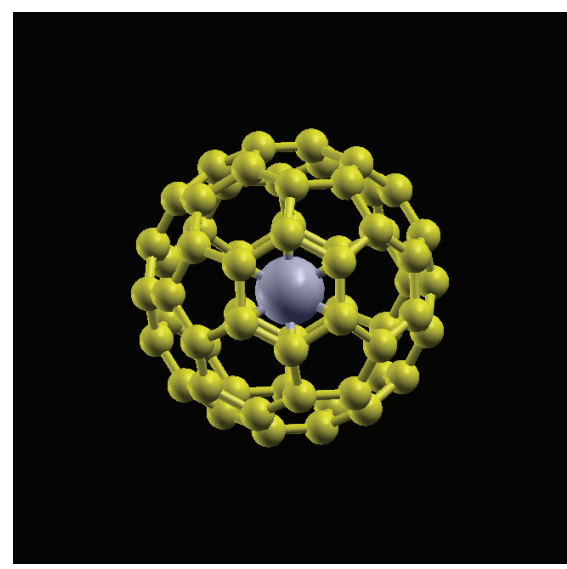

(b)

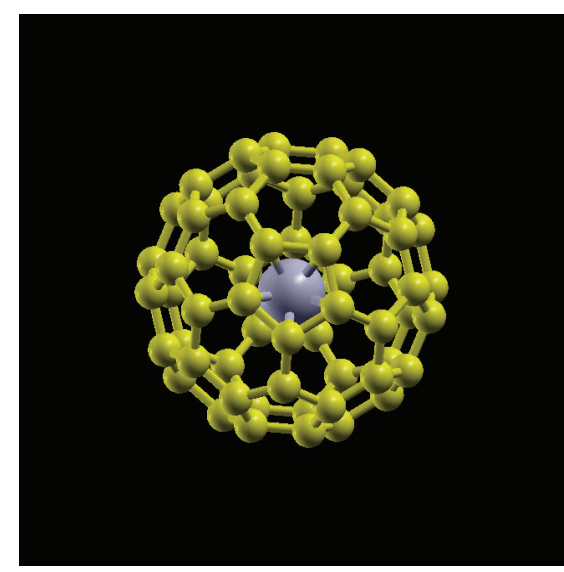

(c)

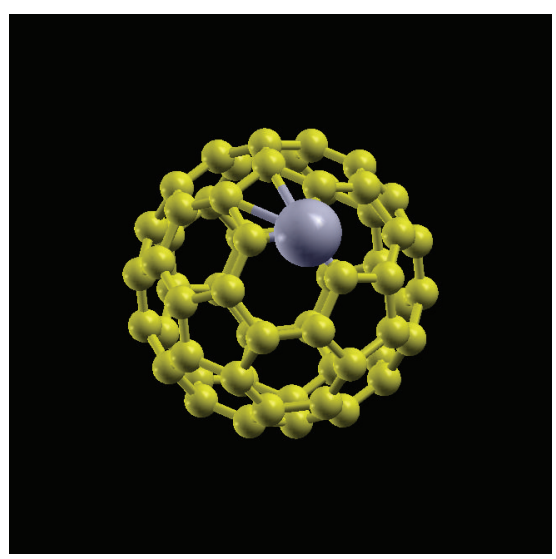

(d)

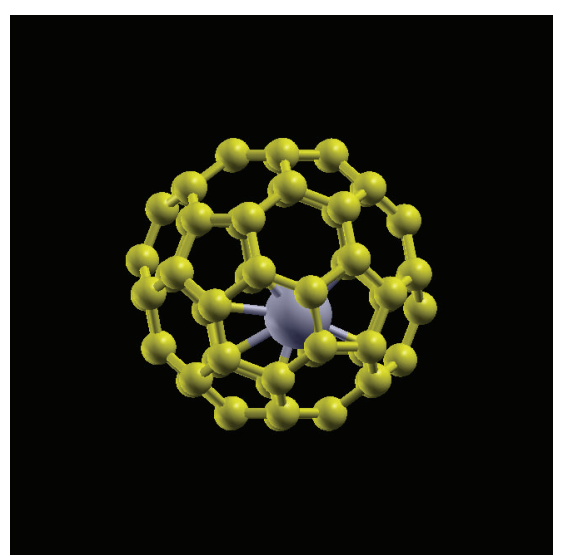

(e)

Figure 6: Fully relaxed configurations of the proposed atomic geometries for the $\mathrm{CeC}_{60}$ with the lowest-energy structure being depicted in (b).

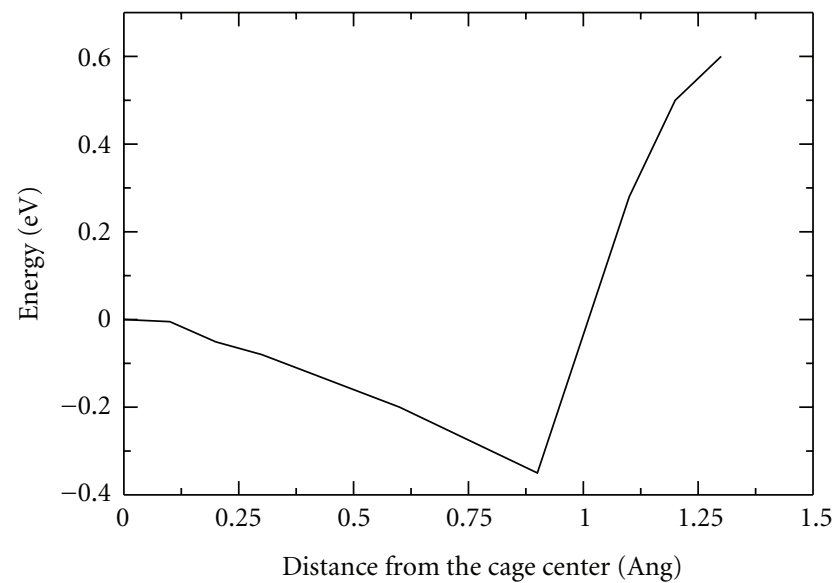

Figure 7: The change of the total energy as a function of the Ce position with respect to the cage center. The energy is set to zero when the Ce atom occupies the cage center.

straight along the $\Gamma \mathrm{X}$ direction, indicating that this state originates from the Ce-filled orbitals. Similarly, the lowest unoccupied state is rather straight along the LX direction,

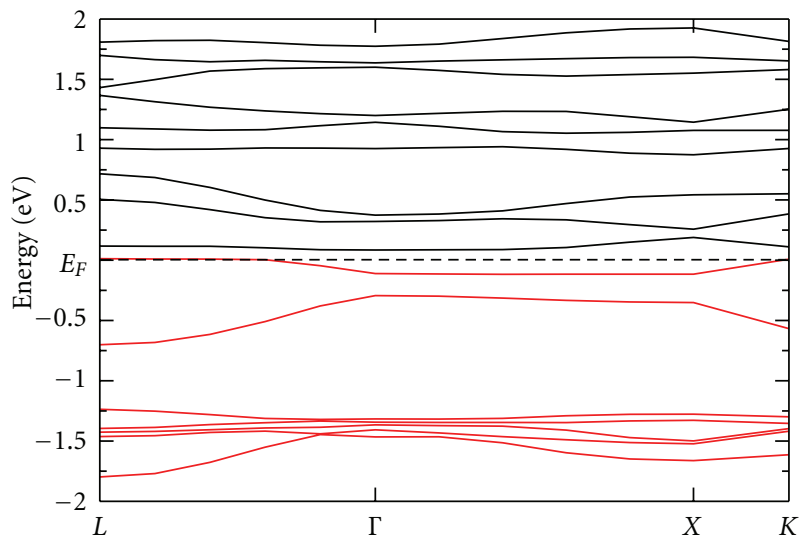

FIGURE 8: The electronic band structure of the Ce-doped fullerene along the principal directions of the Brillouin zone. The Fermi level is set at the zero-energetic position.

suggesting a long-range interaction between the dopant and the host material.

To investigate the density distribution of the present system, we have plotted DOS. Figure 9 shows that the overall 


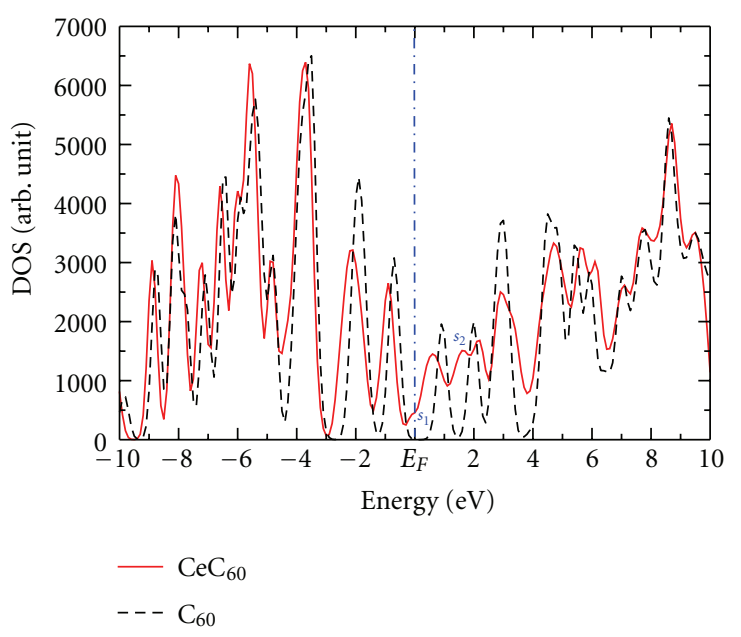

Figure 9: The total density of states (DOSs) of the Ce-doped fullerene. For comparison, the DOS of the fullerene is also depicted. The zero-energetic position refers to the Fermi level.

electronic structure of the system is metallic. Although there are no appreciable changes in the valence region of the Ce-doped fullerene comparing with the clean fullerene, significant changes can be clearly observed in the energy interval $E_{F}$ to $E_{F}+2.5 \mathrm{eV}$. A new shoulder $\mathrm{S}_{1}$ has been created in the vicinity of the fullerene band gap and slightly below the Fermi level. This shoulder, however, is believed to be originated from the $5 d$ orbital of the Ce atom. A well-developed peak is also observed at about $E_{F}+1.8 \mathrm{eV}$, which is partially contributed by the $f$ states of the Ce atom. At approximately $E_{F}+5.0 \mathrm{eV}$, the undeveloped peak observed for fullerene has now been a well-defined peak. Overall, the narrow peaks seen in pure fullerene are now slightly broadened upon the doping of the Ce atom with minor contribution from Ce $4 f$ electrons to the metallic ground state of the system.

To elucidate the nature of the $\mathrm{Ce}-\mathrm{C}$ bonding formation, we have plotted the total charge density along a line joining the Ce atom with its nearest neighbors. Our results, plotted in Figure 10, indicate that the charge is strongly localized around the $\mathrm{C}$ atoms with little amount of charge being accommodated around the dopant, suggesting an ionic nature for the $\mathrm{C}-\mathrm{Ce}$ bond. We also conclude that a large amount of charge has been transferred from the Ce atom to its nearest neighbor $\mathrm{C}$ atoms due to its electronegativity. To estimate the value of the transferring charges, we employed a simple scheme in which we calculate total charge of the total charge density in a sphere, around a Ce atom, of radius equivalent to half the distance between the $\mathrm{Ce}$ atom and the nearest $\mathrm{C}$ atom. Following a similar method with the exact size of the studied unit cell (fullerene cell), the charge density around an isolated Ce atom is calculated. Using the numerical results from these two calculations, approximately $0.64 \mathrm{e}$ charge has been transferred from the $\mathrm{Ce}$ atom to its neighboring $\mathrm{C}$ atoms. The Ce-to- $\mathrm{C}_{60}$ charge transfer, therefore, is responsible for an almost perfect alignment between $(6,6)$ and $(6,5) \mathrm{C}-\mathrm{C}$ bonds.

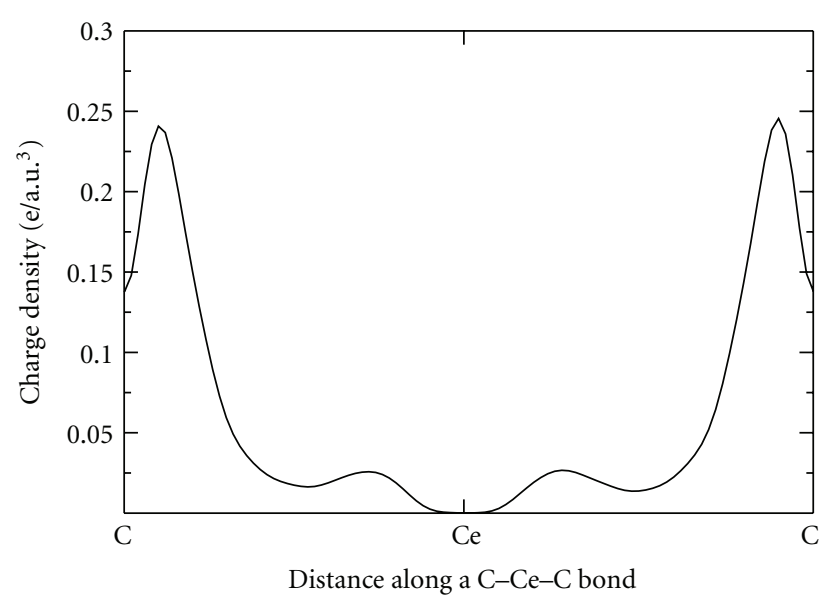

Figure 10: The total charge density along a $\mathrm{C}-\mathrm{Ce}-\mathrm{C}$ bond of the Ce-doped fullerene structure.

TABle 1: A comparison between the Ce and some other metaldoped fullerene is presented.

\begin{tabular}{lcccc}
\hline Metal $\mathrm{C}_{60}$ & $\begin{array}{c}\text { Metal-C } \\
(\AA)\end{array}$ & $\begin{array}{c}\text { Double C-C } \\
(\AA)\end{array}$ & $\begin{array}{c}\text { Single C-C } \\
(\AA)\end{array}$ & $\begin{array}{c}\text { Energy gap } \\
(\mathrm{eV})\end{array}$ \\
\hline $\mathrm{Ce}$ & 2.52 & 1.4115 & 1.467 & 0.2 \\
$\mathrm{Po}$ & & 1.4 & 1.455 & 1.09 \\
$\mathrm{Be}$ & & 1.3943 & 1.4626 & 1.25 \\
$\mathrm{Ca}$ & & 1.4082 & 1.465 & 0.5 \\
$\mathrm{Ni}$ & 1.805 & 1.43 & 1.46 & \\
\hline
\end{tabular}

A summary of the structural and electronic parameters of $\mathrm{CeC}_{60}$ compared with other endohedral metal-doped $\mathrm{C}_{60}$ is presented in Table 1.

\section{Summary}

Using $a b$ initio density functional calculations, the structural and electronic properties of Ce-doped fullerene have been investigated. It is found that the ground-state structure of $\mathrm{CeC}_{60}$ composes of a $\mathrm{Ce}$ atom bonded to the hexagonal ring of the cage with a binding energy of approximately $5.34 \mathrm{eV}$. The structure is semiconducting at the $\Gamma$ point with a significant reduction in the fullerene band gap. The $\mathrm{C}-\mathrm{Ce}$ bond is found to be of an ionic nature with a large amount of charge being transferred from the Ce to the $\mathrm{C}$ atoms. Upon the charge transfer from the $\mathrm{Ce}$ to $\mathrm{C}$ atoms, the double and single $\mathrm{C}-\mathrm{C}$ bonds have been slightly elongated.

\section{Acknowledgment}

The financial support (project no. 66/130/1431) from the deanship of scientific research (DSR) at King Abdulaziz University (KAU) is highly appreciated and gratefully acknowledged. 


\section{References}

[1] H. W. Kroto, J. R. Heath, S. C. O’Brien, R. F. Curl, and R. E. Smalley, " $\mathrm{C}_{60}$ : buckminsterfullerene," Nature, vol. 318, no. 6042, pp. 162-163, 1985.

[2] S. Iijima, "Helical microtubules of graphitic carbon," Nature, vol. 354 , no. 6348 , pp. 56-58, 1991.

[3] K. S. Novoselov, A. K. Geim, S. V. Morozov et al., "Electric field in atomically thin carbon films," Science, vol. 306, no. 5696, pp. 666-669, 2004.

[4] T. A. Spurlin and A. A. Gewirth, "Effect of $\mathrm{C}_{60}$ on solid supported lipid bilayers," Nano Letters, vol. 7, no. 2, pp. 531-535, 2007.

[5] D. Boawan, N. Thomwattana, and J. M. Hill, "Encapsulation of $\mathrm{C}_{60}$ fullerenes into single-walled carbon nanotubes: fundamental mechanical principles and conventional applied mathematical modeling," Physical Review B, vol. 76, no. 15, Article ID 155411, 8 pages, 2007.

[6] Q. Wang, "Torsional instability of carbon nanotubes encapsulating $\mathrm{C}_{60}$ fullerenes," Carbon, vol. 47 , no. 2, pp. 507-512, 2009.

[7] S. Yang, S. Guo, S. Bai, E. Khosravi, G. L. Zhao, and D. Bagayoko, "Doped $\mathrm{C}_{60}$ study from first principles simulation," Journal of Superconductivity and Novel Magnetism, vol. 23, no. 6, pp. 877-880, 2010.

[8] S. Saito and A. Oshiyama, "Cohesive mechanism and energy bands of solid $\mathrm{C}_{60}$," Physical Review Letters, vol. 66, no. 20, pp. 2637-2640, 1991.

[9] J. R. Heath, S. C. O’Brien, Q. Zhang et al., "Lanthanum complexes of spheroidal carbon shells," Journal of the American Chemical Society, vol. 107, no. 25, pp. 7779-7780, 1985.

[10] H. Shinohara, H. Sato, Y. Saito, M. Ohkohchi, and Y. Ando, "Mass spectroscopic and ESR characterization of soluble yttrium-containing metallofullerenes $\mathrm{YC}_{82}$ and $\mathrm{Y}_{2} \mathrm{C}_{82}$," Journal of Physical Chemistry, vol. 96, no. 9, pp. 3571-3573, 1992.

[11] H. Shinohara, H. Sato, M. Ohkohchi et al., "Encapsulation of a scandium trimer in $\mathrm{C}_{82}$," Nature, vol. 357, no. 6373, pp. 52-54, 1992.

[12] A. R. Kortan, N. Kopylov, S. Glarum et al., "Superconductivity at $8.4 \mathrm{~K}$ in calcium-doped $\mathrm{C}_{60}$ " Nature, vol. 355 , no. 6360, pp. 529-532, 1992.

[13] T. Ohtsuki, K. Masumoto, K. Ohno et al., "Insertion of Be atoms in $\mathrm{C}_{60}$ fullerene cages: $\mathrm{Be} \mathrm{C}_{60}$," Physical Review Letters, vol. 77, no. 17, pp. 3522-3524, 1996.

[14] Y. Kubozono, H. Maeda, Y. Takabayashi et al., "Isolation and characterization of $\mathrm{C}_{60}$," Journal of the American Chemical Society, vol. 118, no. 29, pp. 6998-6999, 1996.

[15] T. Inoue, Y. Kubozono, S. Kashino et al., "Electronic structure of Eu@C $\mathrm{C}_{60}$ studied by XANES and UV-VIS absorption spectra," Chemical Physics Letters, vol. 316, no. 5-6, pp. 381-386, 2000.

[16] T. Ogawa, T. Sugai, and H. Shinohara, "Isolation and characterization of Er@ $\mathrm{C}_{60}$," Journal of the American Chemical Society, vol. 122, no. 14, pp. 3538-3539, 2000.

[17] A. Gromov, N. Krawez, A. Lassesson, D. I. Ostrovskii, and E. E. B. Campbell, "Optical properties of endohedral Li@ $\mathrm{C}_{60}$," Current Applied Physics, vol. 2, no. 2, pp. 51-55, 2002.

[18] R. Klingeler, C. Breuer, I. Wirth et al., "Scanning tunneling spectroscopy of small Ce-doped endohedral fullerenes on HOPG," Surface Science, vol. 553, no. 1-3, pp. 95-104, 2004.

[19] W. Y. Ching, M. Z. Huang, Y. N. Xu, W. G. Harter, and F. T. Chan, "First-principles calculation of optical properties of $\mathrm{C}_{60}$ in the fcc lattice," Physical Review Letters, vol. 67, no. 15, pp. 2045-2048, 1991.
[20] Y. N. Xu, M. Z. Huang, and W. Y. Ching, "Optical properties of superconducting $\mathrm{K}_{3} \mathrm{C}_{60}$ and insulating $\mathrm{K}_{6} \mathrm{C}_{60}$," Physical Review $B$, vol. 44, no. 23, pp. 13171-13174, 1991.

[21] S. Saito and A. Oshiyama, "Electronic structure of calciumdoped $\mathrm{C}_{60}$," Solid State Communications, vol. 83, no. 2, pp. 107-110, 1992.

[22] K. Umemoto and S. Saito, "Electronic structure of the $\mathrm{Ba}_{4} \mathrm{C}_{60}$ superconductor," Physical Review B, vol. 61, no. 20, pp. 1420414208, 2000.

[23] J. Lu, Y. Zhou, X. Zhang, and X. Zhao, "Density functional theory studies of beryllium-doped endohedral fullerene Be@ $\mathrm{C}_{60}$ : on center displacement of beryllium inside the $\mathrm{C}_{60}$ cage," Chemical Physics Letters, vol. 352, no. 1-2, pp. 8-11, 2002.

[24] Ş. Erkoç and L. Türker, "Structural and electronic properties of endofullerenes X@ $\mathrm{C}_{60}$," Journal of Molecular Structure, vol. 634, pp. 195-199, 2003.

[25] Ş. Erkoç and L. Türker, "AM1 treatment of endohedrally hydrogen doped fullerene, $n \mathrm{H}_{2} @ \mathrm{C}_{60}$," Journal of Molecular Structure, vol. 638, pp. 37-40, 2003.

[26] Ş. Erkoç and L. Türker, "Ammonia deposition in fullerene: $\left(\mathrm{NH}_{3}\right) n @ \mathrm{C}_{60}$," Journal of Molecular Structure, vol. 640, pp. 5761, 2003.

[27] T. Ohtsuki and K. Ohno, "Formation of Po@ $\mathrm{C}_{60}$," Physical Review B, vol. 72, no. 15, Article ID 153411, 3 pages, 2005.

[28] H. Dodziuk, "Modeling complexes of $\mathrm{H}_{2}$ molecules in fullerenes," Chemical Physics Letters, vol. 410, no. 1-3, pp. 39-41, 2005.

[29] M. Chi, P. Han, X. Fang, W. Jia, X. Liu, and B. Xu, "Density functional theory of Polonium-doped endohedral fullerenes Po@ $\mathrm{C}_{60}$, Journal of Molecular Structure, vol. 807, no. 1-3, pp. 121-124, 2007.

[30] G. Li, R. F. Sabirianov, J. Lu, X. C. Zeng, and W. N. Mei, "Electronic and magnetic properties of endohedrally doped fullerene Mn@ $\mathrm{C}_{60}$ : a total energy study," Journal of Chemical Physics, vol. 128, no. 7, Article ID 074304, 6 pages, 2008.

[31] I. Amato, "Researchers swap material evidence in Boston," Science, vol. 258, no. 5090, pp. 1886-1887, 1992.

[32] G. Kresse and J. Hafner, "Ab initio molecular dynamics for liquid metals," Physical Review B, vol. 47, no. 1, pp. 558-561, 1993.

[33] G. Kresse and J. Furthmüller, "Efficiency of ab-initio total energy calculations for metals and semiconductors using a planewave basis set," Computational Materials Science, vol. 6, no. 1, pp. 15-50, 1996.

[34] G. Kresse and D. Joubert, "From ultrasoft pseudopotentials to the projector augmented-wave method," Physical Review B, vol. 59, no. 3, pp. 1758-1775, 1999.

[35] G. Kresse and J. Furthmüller, "Efficient iterative schemes for ab initio total-energy calculations using a plane-wave basis set," Physical Review B, vol. 54, no. 16, pp. 11169-11186, 1996.

[36] W. Kohn and L. J. Sham, "Self-consistent equations including exchange and correlation effects," Physical Review A, vol. 140, no. 4, pp. A1133-A1138, 1965.

[37] D. Vanderbilt, "Soft self-consistent pseudopotentials in a generalized eigenvalue formalism," Physical Review B, vol. 41, no. 11, pp. 7892-7895, 1990.

[38] J. P. Perdew and A. Zunger, "Self-interaction correction to density-functional approximations for many-electron systems," Physical Review B, vol. 23, no. 10, pp. 5048-5079, 1981.

[39] H. J. Monkhorst and J. D. Pack, "Special points for Brillouinzone integrations," Physical Review B, vol. 13, no. 12, pp. 51885192, 1976. 
[40] M. S. Dresselhaus, G. Dresselhaus, and P. C. Eklund, Science of Fullerenes and Carbon Nanotubes, Academic Press, San Diego, Calif, USA, 1996.

[41] S. Pekker, E. Kováts, G. Oszlányi et al., "Rotor-stator molecular crystals of fullerenes with cubane," Nature Materials, vol. 4, no. 10, pp. 764-767, 2005.

[42] V. Zólyomi, J. Koltai, J. Kürti, and S. Pekker, "Theoretical study of the electronic structure of fullerene-cubane cocrystals," Physical Review B, vol. 78, no. 11, Article ID 115405, 6 pages, 2008.

[43] W. I. F. David, R. M. Ibberson, J. C. Matthewman et al., "Crystal structure and bonding of ordered $\mathrm{C}_{60}$," Nature, vol. 353, no. 6340, pp. 147-149, 1991.

[44] H. Mauser, N. J. R. V. Hommes, T. Clark et al., "Stabilization of atomic nitrogen inside $\mathrm{C}_{60}$," Angewandte Chemie, vol. 36, no. 24, pp. 2835-2838, 1997.

[45] A. Weidinger, M. Waiblinger, B. Pietzak, and T. Almeida Murphy, "Atomic nitrogen in $\mathrm{C}_{60}: \mathrm{N} @ \mathrm{C}_{60}$," Applied Physics A, vol. 66, no. 3, pp. 287-292, 1998.

[46] L. Pang and F. Brisse, "Endohedral energies and translation of fullerene-noble gas clusters G@Cn ( $\mathrm{G}$ = helium, neon, argon, krypton and xenon; $n=60$ and 70)," Journal of Physical Chemistry, vol. 97, no. 33, pp. 8562-8563, 1997.

[47] M. Son and Y. K. Sung, “The atom-atom potential. Exohedral and endohedral complexation energies of complexes of $\mathrm{X} @ \mathrm{C}_{60}$ between fullerene and rare-gas atoms ( $\mathrm{X}=\mathrm{He}, \mathrm{Ne}, \mathrm{Ar}, \mathrm{Kr}$, and Xe)," Chemical Physics Letters, vol. 245, no. 1, pp. 113-118, 1995.

[48] L. S. Wang, J. M. Alford, Y. Chai et al., "The electronic structure of Ca@C $\mathrm{C}_{60}$," Chemical Physics Letters, vol. 207, no. 4-6, pp. 354-359, 1993.

[49] W. Andreoni and A. Curioni, "Ab initio approach to the structure and dynamics of metallofullerenes," Applied Physics A, vol. 66, no. 3, pp. 299-306, 1998.

[50] W. Andreoni and A. Curioni, "Freedom and constraints of a metal atom encapsulated in fullerene cages," Physical Review Letters, vol. 77, no. 5, pp. 834-837, 1996.

[51] T. Guo, G. K. Odom, and G. E. Scuseria, "Electronic structure of Sc@ $\mathrm{C}_{60}$ : an ab initio theoretical study," Journal of Physical Chemistry, vol. 98, no. 32, pp. 7745-7747, 1994.

[52] E. Neyts, A. Maeyens, G. Pourtois, and A. Bogaerts, "A densityfunctional theory simulation of the formation of $\mathrm{Ni}$-doped fullerenes by ion implantation," Carbon, vol. 49, no. 3, pp. 1013-1017, 2011. 

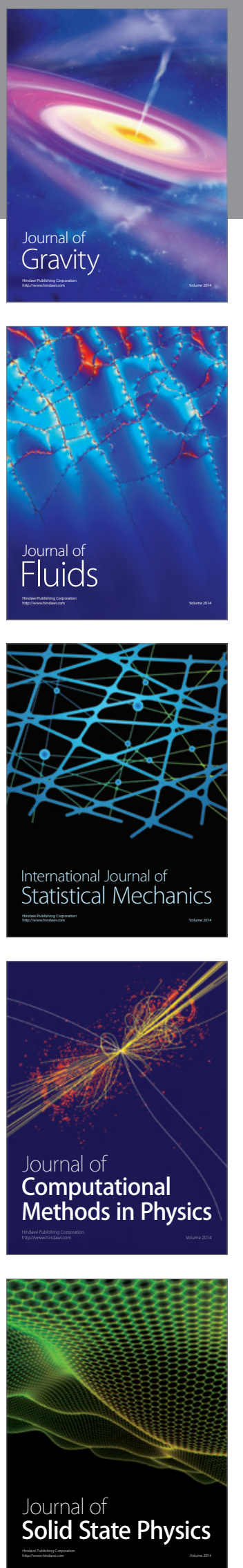
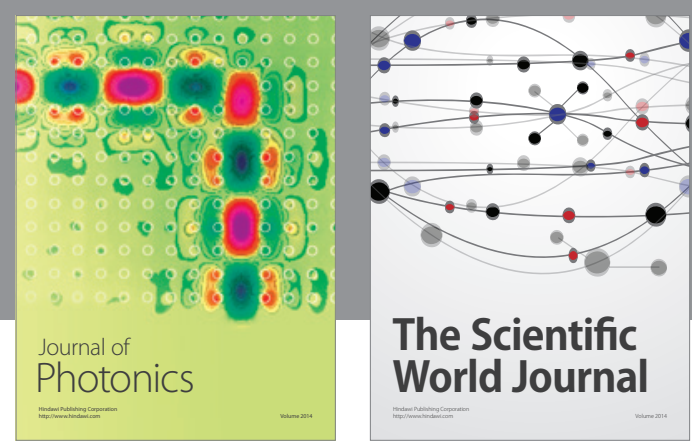

The Scientific World Journal

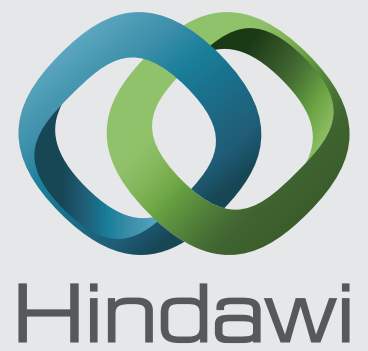

Submit your manuscripts at http://www.hindawi.com
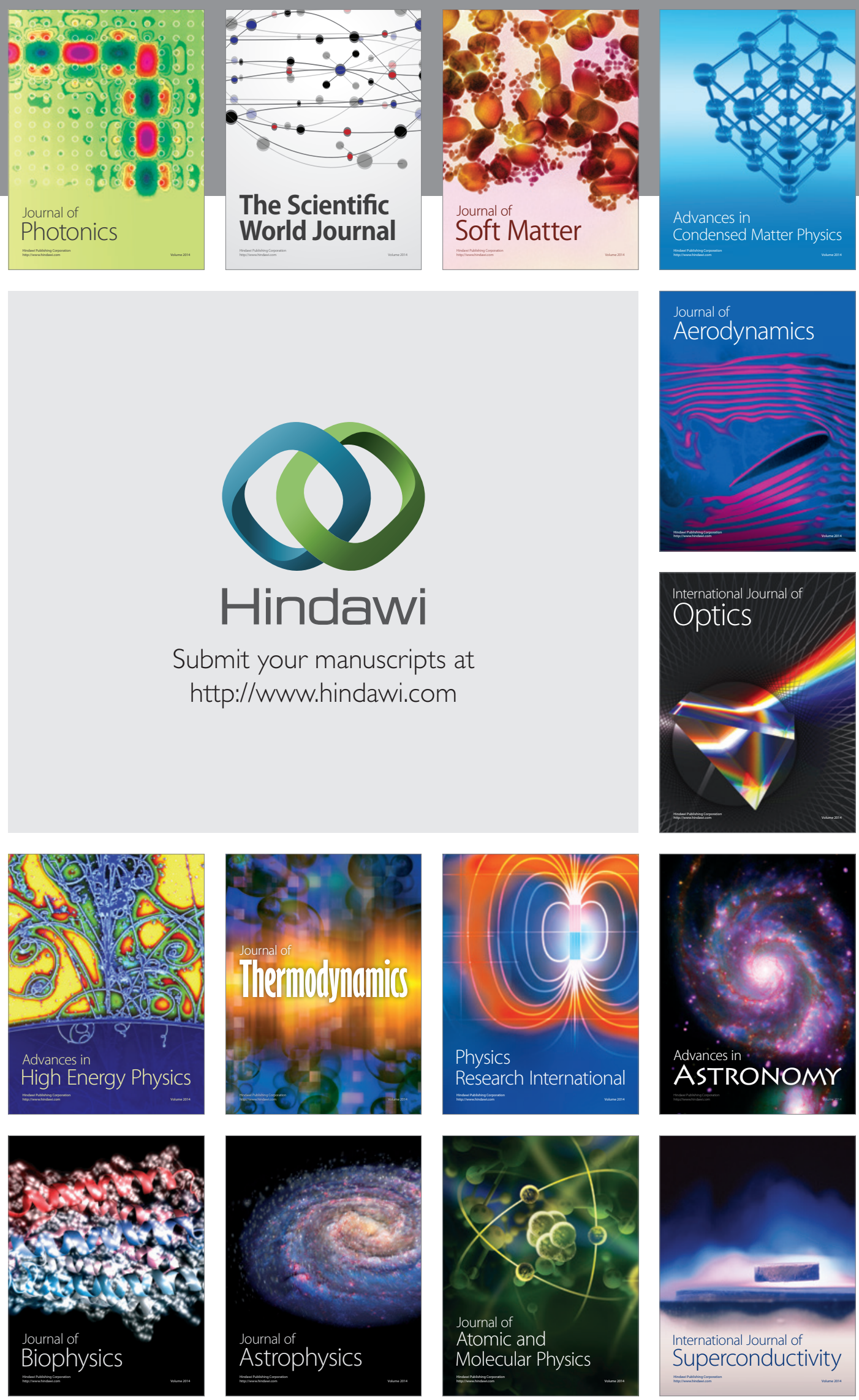
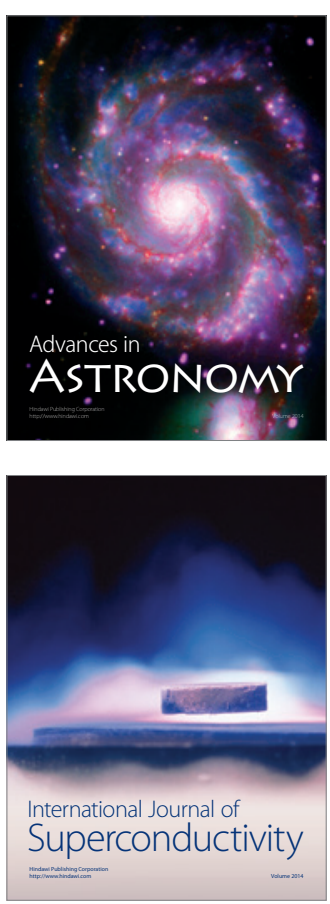